\title{
Impact of clinical and personal data in the dermoscopic differentiation between early melanoma and atypical nevi
}

\author{
Linda Tognetti ${ }^{1,2}$, Elisa Cinotti ${ }^{1}$, Elvira Moscarella ${ }^{3,4}$, Francesca Farnetani ${ }^{5}$, Josep Malvehy ${ }^{6}$, \\ Aimilios Lallas ${ }^{7}$, Giovanni Pellacani ${ }^{5}$, Giuseppe Argenziano ${ }^{3,4}$, \\ Gabriele Cevenini ${ }^{2}$, Pietro Rubegni ${ }^{1}$
}

\begin{abstract}
1 Dermatology Division, Department of Medical, Surgical and NeuroSciences, University of Siena, Siena, Italy 2 Department of Medical Biotechnologies, University of Siena, Siena, Italy 3 Skin Cancer Unit, Arcispedale Santa Maria Nuova, IRCCS, Reggio Emilia, Italy

4 Dermatology Unit, University of Campania, Naples, Italy

5 Department of Dermatology, University of Modena and Reggio Emilia, Modena Italy 6 Melanoma Unit, Department of Dermatology, University of Barcelona, Barcelona, Spain

7 First Department of Dermatology, Aristotele University, Thessaloniki, Greece
\end{abstract}

Key words: melanoma, atypical nevi, dermoscopy, clinical and personal data

Citation: Tognetti L, Cinotti E, Moscarella E, Farnetani F, Malvehy J, Lallas A, Pellacani G, Argenziano G, Cevenini G, Rubegni P. Impact of clinical and personal data in the dermoscopic differentiation between early melanoma and atypical nevi. Dermatol Pract Concept. 2018;8(4):324-327. DOI: https://doi.org/10.5826/dpc.0804a16

Received: March 26, 2018; Accepted: May 23, 2018; Published: October 31, 2018

Copyright: (92018 Tognetti et al. This is an open-access article distributed under the terms of the Creative Commons Attribution License, which permits unrestricted use, distribution, and reproduction in any medium, provided the original author and source are credited.

Funding: None.

Competing interests: The authors have no conflicts of interest to disclose.

All authors have contributed significantly to this publication. LT and PR contributed equally to this study.

Corresponding author: Linda Tognetti, MD, Hospital S. Maria alle Scotte, Viale Bracci 16, 53100 Siena, Italy. Email: l.tognetti@ studentunisi.it

ABSTRACT Background: Differential diagnosis of clinically atypical nevi (aN) and early melanomas (eMM) still
represents a challenge even for experienced dermoscopists, as dermoscopy alone is not sufficient to
adequately differentiate these equivocal melanocytic skin lesions (MSLs).
Objectives: The objectives of this study were to investigate what were the most relevant parameters
for noninvasive differential diagnosis between eMM and aN among clinical, personal, and dermo-
scopic data and to evaluate their impact as risk factors for malignancy.
Methods: This was a retrospective study performed on 450 MSLs excised from 2014 to 2016 with a
suspicion of malignancy. Dermoscopic standardized images of the 450 MSLs ( 300 aN and 150 eMM)
were collected and evaluated. Patients' personal data (ie, age, gender, body site, maximum diameter)
were also recorded. Dermoscopic evaluations were performed by 5 different experts in dermoscopy
blinded to histopathological diagnosis. Fleiss' $\kappa$ was calculated to measure concordance level between
experts in the description of dermoscopic parameters for each MSL. The power of the studied vari-
ables in discriminating malignant from benign lesions was also investigated through F-statistics. 
ABSTRACT Results: The variables age and maximum diameter supplied the highest discriminant power $(F=$ 253 and 227, respectively). Atypical network, blue white veil and white shiny streaks were the most significant dermoscopic patterns suggestive of malignancy $(F=110,104$ and 99.5 , respectively). Shiny white streaks was the only dermoscopic parameter to obtain satisfactory concordance value. Gender was not a discriminant factor. The specific statistical weight of clinical and personal data (ie, "patient's age" and "lesion diameter") surpassed those of atypical dermoscopic features.

Conclusions: The objective clinical and personal data collected here could supply a fundamental contribution in the correct diagnosis of equivocal MSLs and should be included in diagnostic algorithms along with significant dermoscopic features (ie, atypical network, blue-white veil, and shiny white streaks).

\section{Introduction}

Dermoscopy is a useful noninvasive diagnostic method for differentiating benign from malignant melanocytic skin lesions (MSLs) [1]. In clinical practice, equivocal MSLs, including early melanomas (eMM), that do not yet exhibit clear-cut atypical features and atypical nevi $(\mathrm{aN})$ showing clinical and dermoscopic features usually associated with malignancy are seen frequently. Early diagnosis of these equivocal MSLs can be challenging even for experienced dermoscopists [2-5]. In daily practice, dermatologists consider a patient's risk factors that together form a basis for the decision "to leave or to excise" that include lesion dimension, localization, evolution in time, number of nevi, personal/familial history of melanoma, and skin phototype [6-8]. However, only 4 criteria-body site, maximum diameter, age, and sex-represent objective and standardized variables to assess for malignancy.

The objective of this study was to define which clinical and personal data are the most relevant risk factors for malignancy and to investigate their impact in the dermoscopic differential diagnosis between eMM and aN.

\section{Methods}

A total of 493 atypical MSLs were excised from 2014 to 2016 with suspected malignancy (Figure 1). MSLs localized on the face, palms, and soles were excluded a priori due to their pecu-
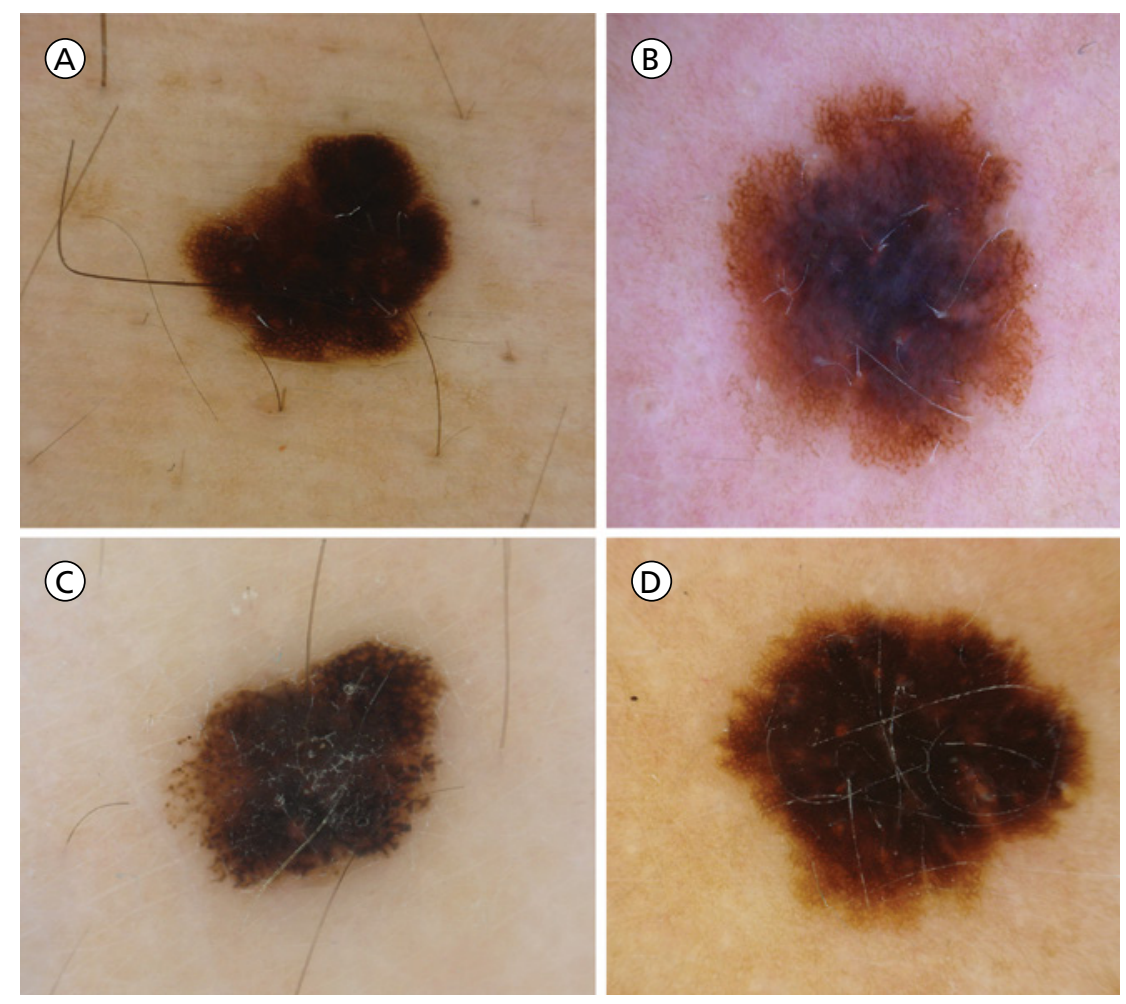

Figure 1. Examples of dermoscopically and clinically equivocal MSLs from the case study (polarized dermoscopy, 20x) diagnosed histologically. Atypical nevi exhibiting atypical network (A, B), blue-white veil and shiny white streaks (B). Early melanomas (C, D) showing only irregular dots and globules $(C)$ and irregular pigmented blothes (D). Nevi were excised from the abdomen of a 43-year-old woman (A) and the arm of a 51-year-old man (B). Melanomas were excised from the upper back of an 83-year-old man (C) and a 79-year-old woman (D).

liar dermoscopic pattern. After selection for image quality, availability of patient data, and agreement of $3 / 3$ experts on histopathological diagnosis, the final database consisted of 450 standardized dermoscopic micrographs-300 aN and $150 \mathrm{eMM}$ - acquired at $17 \times$ magnification. Dermoscopic evaluations were independently performed by 5 experts in dermoscopy. They were asked to assess the presence/absence of a series of 18 dermoscopic structures designed to include only the features most commonly associated with atypical MSLs according to the current in literature. To ensure a thorough, blinded pattern recognition analysis, all experts were unaware of the histopathological diagnosis, clinical and personal data. Then, each one of the 18 selected dermoscopic structures was defined as absent/present within a lesion when $5 / 5$ experts agreed. 
TABLE 1. Discriminant analysis showing F-statistics $(F)$ and $P$-value $(P)$ of all dermoscopic, clinical, and personal variables (47) coded into 38 simple variables, 5 whole variables (bold), and 4 grouped variables (italics)

\begin{tabular}{|c|c|c|c|c|c|}
\hline Integrated Variables & $\boldsymbol{F}$ & $p$ & Integrated Variables & $\boldsymbol{F}$ & $p$ \\
\hline Age (years) & 253 & .000 & Atypical vascular pattern (AVP) & 21.2 & .000 \\
\hline Maximum Diameter (mm) & 227 & .000 & Shoulders & 13.7 & .000 \\
\hline Age cut-off $\geq 40$ years & 197 & .000 & Group C: Seldom exposed sites & 13.5 & .000 \\
\hline Age cut-off $\geq 60$ years & 167 & .000 & Hypopigmented areas & 13.4 & .000 \\
\hline Maximum diameter cut-off $\geq 8$ & 152 & .000 & Blue-gray globules & 13.3 & .000 \\
\hline Age cut-off $\geq 50$ years & 146 & .000 & Arms + hands & 12.6 & .000 \\
\hline Maximum diameter cut-off $\geq 5$ & 133 & .000 & Group A: Chronically exposed sites & 12.1 & .001 \\
\hline Maximum diameter cut-off $\geq 7 \mathrm{~mm}$ & 129 & .000 & Group D: Rarely exposed sites & 9.73 & .002 \\
\hline Maximum diameter cut-off $>10 \mathrm{~mm}$ & 124 & .000 & Back & 7.21 & .007 \\
\hline Atypical network & 110 & .000 & Anatomical Site & 6.82 & .009 \\
\hline Age cut-off $\geq 30$ years & 108 & .000 & Neck & 6.78 & .009 \\
\hline Blue-white veil & 104 & .000 & Chest/breast & 5.68 & .017 \\
\hline Shiny white streaks & 99.5 & .000 & Ankle + back of the feet & 5.47 & .019 \\
\hline Irregular pigmented blotches & 67.6 & .000 & Side & 4.35 & .037 \\
\hline Irregular streaks & 55.6 & .000 & Head & 2.69 & .102 \\
\hline Pink areas & 52.3 & .000 & Multicolor pattern & 2.68 & .06 \\
\hline Blue-white veil >30\% & 47.6 & .000 & Broad network & 2.57 & .08 \\
\hline White scar-like areas & 41.1 & .000 & Bottom & 2.55 & .110 \\
\hline Blue-gray peppering & 39.2 & .000 & Abdomen & 2.30 & .130 \\
\hline UV-exposed Body Areas & 39.2 & .000 & Radial streaming & 2.6 & .10 \\
\hline Group B: Frequently exposed sites & 27.9 & .000 & Multiple brown dots & 2.3 & .12 \\
\hline Legs & 23.5 & .000 & Light brown areas & 2.1 & .13 \\
\hline Irregular dots and globules (IDG) & 22.6 & .000 & Gender & 2.1 & .28 \\
\hline
\end{tabular}

Overall interobserver agreement was estimated by Fleiss' $\kappa$ and its $95 \%$ confidence interval (CI). In a second phase, we retrospectively collected 2 clinical data (diameter and body site) and 2 personal data (age and sex) sets for each of the 450 MSLs, obtaining an integrated database of 450 images associated with 18 subjective (ie, dermoscopic data) and 4 objective variables (ie, clinical-personal data). In order to be tested for risk factors for malignancy, they were evaluated both in their original form as 5 whole variables and in their binary-coded form as 42 simple variables. Age and maximum diameter were dichotomized to account for some interesting cut-off values. The lesion site was described according to ana- tomical criteria and further grouped into 4 body areas according to UV exposure, ie, Group A, chronically photoexposed body sites (head, neck, arms/ hands); Group B, frequently photoexposed body sites (thighs, legs, ankle, back of the feet); Group C, seldom photoexposed body sites (shoulders, chest/breast, back); and Group D, rarely photoexposed body sites (abdomen, bottom, side).

\section{Results}

Univariate discriminant analysis of all 47 integrated variables, shown in Table 1, was performed taking the histopathological diagnosis as outcome. Univariate power to discriminate between
eMM and aN was quantified by means of F-statistics. Statistical significance $(P<0.05)$ was obtained by $37 / 47$ variables. Taken together, the results of this analysis showed that: 1) age and diameter exhibited the highest discriminant power for eMM when considered as whole or simple variables; 2) the classification of anatomical sites into 4 body area groups according to UV exposure resulted in association with malignancy (eg, body site "head" obtained $P>0.05$ and $F<2.69$ as simple variable, but $P<0.05$ and $F=12.1$ when as part of Group A, chronically exposed body areas); 3) none of the dermoscopic features reached $F>110$, demonstrating moderate impact; and 4) as reported in Table 2, agreement between experts 
TABLE 2. Concordance levels of experts $\left(D_{1}-D_{5}\right)$ in recognition of dermoscopic structures (only variables that obtained $P>0.05$ are shown)

\begin{tabular}{|l|c|c|}
\hline \multicolumn{1}{|c|}{ Dermoscopic Variable } & Fleiss' $\mathbf{k}[\mathbf{9 5} \% \mathbf{~ C l ]}$ & Strength of Agreement \\
\hline Shiny white streaks & $0.418[0.403-0.432]$ & Intermediate \\
\hline Irregular dots and globules & $0.375[0.360-0.389]$ & Poor \\
\hline Blue-white veil & $0.334[0.320-0.349]$ & Poor \\
\hline Blue-gray globules & $0.302[0.287-0.316]$ & Poor \\
\hline Hypopigmented areas & $0.297[0.283-0.312]$ & Poor \\
\hline Irregular streaks & $0.254[0.239-0.268]$ & Poor \\
\hline Atypical network & $0.238[0.224-0.253]$ & Poor \\
\hline White scar-like areas & $0.214[0.200-0.229]$ & Poor \\
\hline Pigmented areas & $0.163[0.148-0.177]$ & Poor \\
\hline Blue-white veil $>30$ & $0.151[0.137-0.166]$ & Poor \\
\hline Atypical vascular pattern & $0.100[0.085-0.114]$ & Poor \\
\hline Blue-gray peppering & $0.098[0.084-0.113]$ & Poor \\
\hline Irregular pigmented blotches & $0.077[0.063-0.091]$ & \\
\hline
\end{tabular}

was generally poor, with the exception of white shiny streaks $(\kappa=0.418,95 \%$ CI 0.403-0.432) albeit of intermediate level, which was probably due to the clear-cut appearance of this pattern.

\section{Conclusions}

The method of combining clinical and personal data with dermoscopic variables proved to be highly useful diagnostically in differentiating regressing MM from regressing nevi [8]. Here in this dataset of eMM and aN, the relative impact of dermoscopic structures was moderate and their recognition was confirmed to be a rather subjective and equivocal method with unsatisfactory agreement [1,2]. Our findings are in line with recent epidemiological data in that eMM shows a trend to be increasing in prevalence in the elderly with no gender predominance $[5,6]$, a strong correlation with lesion maximum diameter $[7,8]$, and a moderate correlation with UV exposure ( $F=39.4)$. This probably reflects only a fraction of eMM that develop due to UV exposure [5-7]. In conclusion, despite the contemporary presence of an atypical network, blue-white veil, and shiny white streaks within an equivocal MSLs, which may indicate malignancy, the objective clinical and personal data collected could supply a fundamental contribution in the correct diagnosis of equivocal MSLs and should be included in diagnostic algorithms.

\section{References}

1. Argenziano G, Cerroni L, Zalaudek I, et al. Accuracy in melanoma detection: a 10-year multicenter survey. J Am Acad Dermatol. 2012;67(1):54-59.

2. Carrera C, Marchetti MA, Dusza SW, et al. Validity and reliability of dermoscopic criteria used to differentiate nevi from melanoma: a web-based international dermoscopy society study. JAMA Dermatol. 2016;152(7):798-806.

3. Abbasi NR, Yancovitz M, GutkowiczKrusin D, et al. Utility of lesion diameter in the clinical diagnosis of cutaneous mela- noma. Arch Dermatol. 2008;144(4):469474.

4. Moreno-Ramírez D, Ojeda-Vila T, RíosMartín JJ, et al. Association between tumor size and Breslow's thickness in malignant melanoma: a cross-sectional, multicenter study. Melanoma Res. 2015;25(5): 450-452.

5. Haenssle HA, Mograby N, Ngassa A, et al. Association of patient risk factors and frequency of nevus-associated cutaneous melanomas. JAMA Dermatol. 2016;152(3):291-298.

6. Whiteman DC, Green AC, Olsen CM. The growing burden of invasive melanoma: projections of incidence rates and numbers of new cases in six susceptible populations through 2031. J Invest Dermatol. 2016;136(6):1161-1171.

7. Liu F, Bessonova L, Taylor TH, et al. A unique gender difference in early onset melanoma implies that in addition to ultraviolet light exposure other causative factors are important. Pigment Cell Melanoma Res. 2013; 26(1):128-135.

8. Rubegni P, Tognetti L, Argenziano G, et al. A risk scoring system for the differentiation between melanoma with regression and regressing nevi. J Dermatol Sci. 2016; 83(2):138-144. 